\title{
Propagation of surface acoustic waves through sharply bent two-dimensional phononic crystal waveguides using a finite-difference time-domain method
}

\author{
Jia-Hong Sun and Tsung-Tsong Wu* \\ Ultrasonics Lab., Institute of Applied Mechanics, National Taiwan University, Taipei 106, Taiwan \\ (Received 26 June 2006; revised manuscript received 1 September 2006; published 30 November 2006)
}

\begin{abstract}
In this paper, we adopt the finite-difference time-domain (FDTD) method to analyze surface acoustic waves propagating in two-dimensional phononic waveguides. To implement the FDTD program for dealing with surface acoustic waves, the Bloch theorem and absorbing boundary conditions are employed to deal with the periodic boundary condition and reflection from a numerical boundary. A phononic crystal consisting of circular steel cylinders that form a square lattice in an epoxy matrix is considered as an example to study phononic crystal waveguides. The dispersion relation and displacement fields are calculated to identify the band gaps and eigenmodes. The result shows the existence of a complete band gap of surface waves and thus an acoustic waveguide is created accordingly. Eigenmodes of surface waves inside the waveguide are indicated and pseudo surface acoustic waves propagating inside the straight waveguide are demonstrated. Further, waveguides with a sharp bend are reported and an improved design is suggested to enhance energy transmission.
\end{abstract}

DOI: 10.1103/PhysRevB.74.174305

PACS number(s): 63.20.-e, 43.20.+g, 43.40.+s, 46.40.Cd

\section{INTRODUCTION}

Phononic crystals consisting of periodic elastic materials have been proved to have a property of band gaps. In various phononic crystals, both the bulk acoustic wave $(\mathrm{BAW})^{1-3}$ and surface acoustic wave (SAW $)^{4-8}$ are analyzed, and complete band gaps, the frequency range that acoustic waves cannot propagate in any direction, were observed in some cases. The complete band gap phenomenon plays an important role in designing phononic crystal applications such as elastic wave filters, couplers, and waveguides, especially. The phononic crystal waveguide is an important elementary component to build an acoustic wave circuit. Currently, most of the phononic waveguide researches are focused on the bulk acoustic waves. The presented BAW waveguides are consisted of solid/solid, ${ }^{9-11}$ solid/liquid, ${ }^{12,13}$ and gas/liquid ${ }^{12}$ phononic crystals. The designs include straight waveguides with various widths, ${ }^{10}$ waveguides with a stub, ${ }^{12}$ a waveguide with hollow cylinders ${ }^{13}$ and a coupler of joined parallel waveguides. ${ }^{11}$ In contrast, the study of SAW in phononic crystal has had less progress. Laude et al. ${ }^{8}$ reported a complete band gap of surface acoustic wave in a piezoelectric phononic crystal; Tanaka and Tamura ${ }^{14}$ analyzed the surface and surface-guided waves in phononic lattices. More investigations on the surface waves in phononic waveguides are awaited.

In this paper, we study the surface wave propagation in phononic waveguides. To accomplish this work, a FDTD method is developed to analyze the dispersion of acoustic wave in phononic crystals. The Bloch theorem and a perfectly matched layer (PML) absorbing boundary condition are employed to deal with the periodic boundary condition and numerical nonreflection boundary respectively. ${ }^{15} \mathrm{~A}$ steel/ epoxy square lattice phononic crystal is studied and the complete band gap of the surface acoustic wave is obtained. With the complete band gap property, a phononic waveguide for surface waves is designed by arranging adjacent point defects. Thus, eigenmodes of surface waves inside the wave- guide are indicated and surface acoustic waves propagating inside the straight waveguide are demonstrated. The result shows that the allowed surface waves can propagate and are confined within the waveguide. Further, the waveguides with a sharp bend are reported and an improved design is suggested to enhance energy transmission.

\section{FINITE-DIFFERENCE TIME-DOMAIN METHOD}

In a linear elastic material, the equation of motion and constitutive law of elastic materials can be expressed as

$$
\begin{gathered}
\rho \ddot{u}_{i}=\tau_{i j, j}+\rho f_{i}, \\
\tau_{i j}=C_{i j k l} \varepsilon_{k l},
\end{gathered}
$$

where $\rho$ is the density of materials, $u_{i}$ the displacement, $\tau_{i j}$ the stress, $f_{i}$ body force, $C_{i j k l}$ elastic constant, and $\varepsilon_{k l}$ strain. Equations (1) and (2) describe the property of an infinitesimal element of an anisotropic material in general. These equations are applicable to the inhomogeneous structure of phononic crystals by arranging the density and elastic constant periodically. Further, with staggered grids, differential equations (1) and (2) are transferred into difference equations based on the Taylor's expansion to develop the threedimensional heterogeneous finite difference formulation. Then these equations are calculated recursively, indefinitely, to study the wave propagation in phononic crystals.

The Bloch's theorem is introduced to treat the periodic boundary condition of a unit cell of phononic crystals. The displacement and stress fields of phononic crystals can be expressed by a plane wave and a periodic function as

$$
\begin{aligned}
& u_{i}(\mathbf{x}, t)=e^{i \mathbf{k} \cdot \mathbf{x}} U_{i}(\mathbf{x}, t), \\
& \tau_{i j}(\mathbf{x}, t)=e^{i \mathbf{k} \cdot \mathbf{x}} T_{i j}(\mathbf{x}, t),
\end{aligned}
$$

where $\mathbf{k}$ is a wave vector. $U_{i}(\mathbf{x}, t)$ and $T_{i j}(\mathbf{x}, t)$ are periodic functions satisfying 


$$
\begin{aligned}
& U_{i}(\mathbf{x}+\mathbf{a}, t)=U_{i}(\mathbf{x}, t), \\
& T_{i j}(\mathbf{x}+\mathbf{a}, t)=T_{i j}(\mathbf{x}, t),
\end{aligned}
$$

with a lattice translation vector a. In Ref. 16, the equation of motion and constitutive law were transformed using the periodic functions to satisfy the conditions (5) and (6). Instead of the transformation, we combined Eqs. (3) and (4) with Eqs. (5) and (6) to write the periodic boundary conditions (PBC) of displacement $u_{i}$ and stress $\tau_{i j}$ directly as ${ }^{17}$

$$
\begin{gathered}
u_{i}(\mathbf{x}+\mathbf{a}, t)=e^{i \mathbf{k} \cdot \mathbf{a}} u_{i}(\mathbf{x}, t), \\
\tau_{i j}(\mathbf{x}+\mathbf{a}, t)=e^{i \mathbf{k} \cdot \mathbf{a}} \tau_{i j}(\mathbf{x}, t) .
\end{gathered}
$$

With the PBC, both the two-dimensional (2D) and threedimensional (3D) phononic crystal cases can be analyzed by calculating a unit cell. In the analyses of dispersions, a small disturbance in a random position of the unit cell is set as the initial condition. Thus, all possible wave modes are transported inside the considered phononic crystal, and the displacement is recorded and expanded into Fourier series. Then the eigenfrequencies of a given wave vector $\mathbf{k}$ are indicated by selecting the resonance peaks of the spectrum, and this allows us to find the information about possible types of bulk waves.

To solve the surface acoustic wave modes of phononic crystals, extra boundary conditions are needed to develop surface acoustic waves inside a phononic crystal unit cell. In this study, we set a free surface boundary condition and use the PML absorbing boundary condition to deal with the reflection from the numerical boundary.

Berenger ${ }^{18}$ introduced the concept of PML to reduce the electromagnetic wave reflection form boundary, and the PML is also developed to calculate the elastic wave propagation. ${ }^{19,20}$ In this paper, a 3D PML program of orthogonal material is adopted to serve the nonreflection boundary condition. A stretched coordinate is defined with a complex variable,

$$
e_{i}=a_{i}+\mathrm{i} \frac{\Omega_{i}}{\omega}
$$

to derive the code of PML region. ${ }^{19}$ In Eq. (9), the real part $a_{i}$ is the scale factor, and $\Omega_{i} / \omega$ is the imaginary part with the attenuation factor $\Omega_{i}$ and the circular frequency $\omega$. Then the differential operation in the stretched coordinate is defined and applied to the equation of motion and constitutive law. After introducing the plane wave solutions into the equations, the numerical attenuation is achieved via the factor $\Omega_{i}$. In addition, a nonreflection condition at the interface between PML region and inner space is obtained by setting corresponding material constants and the unity scale factor. In the elastodynamic equations for the stretched coordinate, displacement and stress components are taken spatial partial differential operation in all directions, thus the variables are split into three components to realize the difference equations. Then actual values are obtained from the summation of splitting components. Finally, the PML equations can be shown, as follows:

$$
\begin{gathered}
\rho \ddot{u}_{i / j}+\rho \Omega_{j} \dot{u}_{i / j}=\tau_{i j, j}, \\
\dot{\tau}_{i j / m}+\Omega_{m} \tau_{i j / m}=C_{i j k l} \frac{\dot{u}_{k, l} \delta_{m l}+\dot{u}_{l, k} \delta_{m k}}{2} .
\end{gathered}
$$

In the above equations, $u_{i / j}$ and $\tau_{i j / m}$ are the splitting displacement and splitting stress, which satisfy $u_{i}=u_{i / 1}+u_{i / 2}$ $+u_{i / 3}$ and $\tau_{i j}=\tau_{i j / 1}+\tau_{i j / 2}+\tau_{i j / 3} . \delta_{i j}$ is the delta function and $\delta_{i j}=1$ when $i=j$, else $\delta_{i j}=0$.

After transforming Eqs. (10) and (11) to difference formula, the PML is arranged outside the space boundaries as a buffer zone with a matched acoustic impedance to suppress the reflection. A numerical attenuation occurs as waves decay rapidly inside the region. With this absorbing boundary condition, the reflection is reduced to less than $1 \%$ and the PML boundary is used in both the calculations of dispersion relation and transmission.

\section{COMPLETE BAND GAPS OF STEEL/EPOXY PHONONIC CRYSTALS}

To investigate the band gap of phononic crystal, the dispersion relation of elastic waves inside phononic crystals is calculated first. In this paper, a square lattice phononic crystal consisting of steel cylinders embedded in an epoxy half space is analyzed to demonstrate the band gap of surface acoustic wave. The lattice constant $a$ of the phononic crystal is $8 \mathrm{~mm}$ and the radius $r$ of steel cylinder is $3 \mathrm{~mm}$, and thus the filling ratio $f$ defined as $\pi r^{2} / a^{2}$ is equal to 0.442 . The density and elastic constant $\mathrm{C}_{11}$ and $\mathrm{C}_{44}$ of steel are assumed as $7900 \mathrm{~kg} / \mathrm{m}^{3}, 280.2 \mathrm{GPa}$, and $82.9 \mathrm{GPa}$, respectively, and those for epoxy are $1180 \mathrm{~kg} / \mathrm{m}^{3}, 7.61 \mathrm{GPa}$, and $1.59 \mathrm{GPa}$. Thus the impedance of cylinders is more than 15 times the one of the matrix.

In $2 \mathrm{D}$ phononic crystal cases, the surface acoustic wave propagates within the $x-y$ plane perpendicular to the cylinders. However, the surface acoustic wave is indeed a $3 \mathrm{D}$ problem in the phononic crystals. We defined a 3D unit cell as shown in Fig. 1(a) to calculate the characteristic mode of surface acoustic waves. The dimension of this unit cell is chosen as $1 a \times 1 a$ of the cross section on the $x-y$ plane and the height $h$ is $9 a$ to develop surface acoustic waves. Then a free surface boundary is on the surface at $z=0$, and a 20layer PML region is under the bottom surface to apply the nonreflection boundary. Finally, the PBC is defined on the other four surfaces of the unit cell. In the FDTD method, each unit cell is divided into $24 \times 24$ grids and a time step interval is $20 \mathrm{~ns}$ to satisfy the numerical stability conditions.

To calculate dispersion curves of acoustic waves, the first Brillouin zone of the square array phononic crystal is shown in Fig. 1(b). The right-angle isosceles triangle refers to the smallest nonreducible area of this phononic crystal, and the vertexes of triangle are denoted as $\Gamma, X$, and $M$, respectively. Similar to the process in calculating the bulk acoustic waves, an initial condition is set and the displacement is recorded and transferred into Fourier series. The eigenmodes are obtained by selecting the resonance peaks on the power spectra. By varying the wave vector $\mathbf{k}$ and repeating the calculation, the dispersion curve is obtained. 


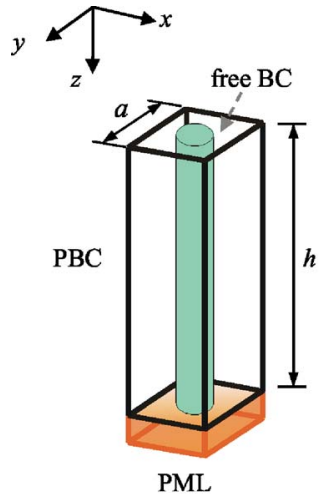

(a)

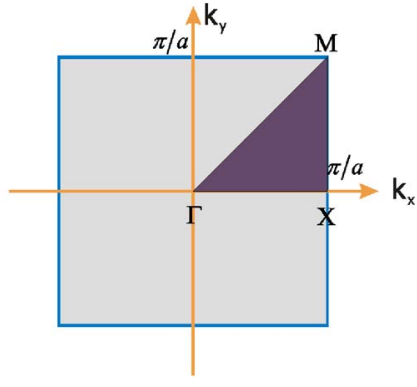

(b)
FIG. 1. (Color online) The phononic crystal with circular rods (steel) embedded in a background material (epoxy) arranged in square lattice. (a) The 3D unit cell of phononic crystal employed to calculate surface acoustic waves; (b) the first Brillouin zone of a square lattice crystal.

In a 2D phononic crystal, the structure is infinite along the $z$ direction. Thus bulk acoustic waves propagate within the $x-y$ plane perpendicular to the cylinders, and the polarization modes are decoupled as the in-plane mode ( $x-y$ plane) and the antiplane mode. In Fig. 2, the eigenmodes of bulk acoustic waves are presented in solid and hollow circles for inplane and antiplane modes respectively. In the in-plane modes, there is a noticeable wide complete band gap from 90 to $204 \mathrm{kHz}$ and several narrow complete band gaps at higher range $231-237 \mathrm{kHz}$ and $245-255 \mathrm{kHz}$. From the curves of antiplane modes, band gaps locate at 55-143, 153212, 225-250, and 270-276 kHz. Considering both modes together, the complete band gaps are at 90-143, 153-204, 231-237, and 245-250 kHz.

Using the 3D unit cell with PBC, free surface, and PML, the eigenmodes are obtained and marked as rhombus symbols in Fig. 2. As shown in the figure, extra modes are detected by the sensors on the free surface. For example, different bands appear at $7-79.5 \mathrm{kHz}$ and $199-201 \mathrm{kHz}$ in the $\Gamma X$ direction. Also, some selected peaks overlap the circle

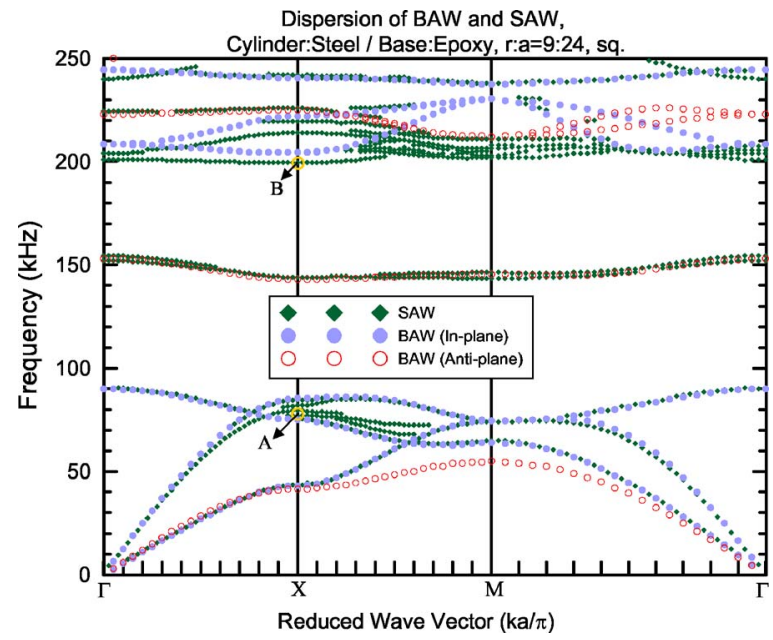

FIG. 2. (Color online) Dispersion of eigenmodes in the square lattice steel/epoxy phononic crystal.

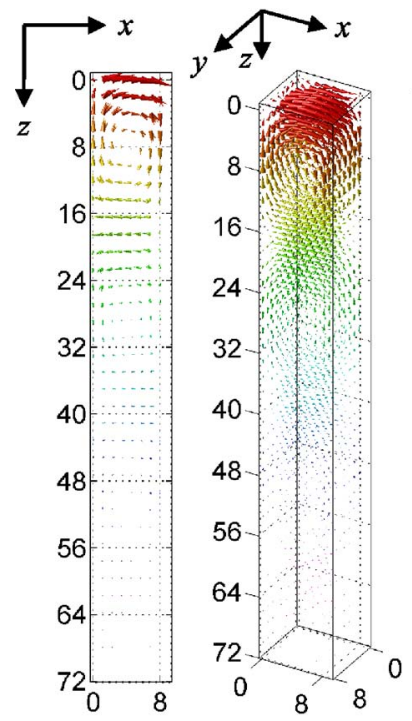

(a)

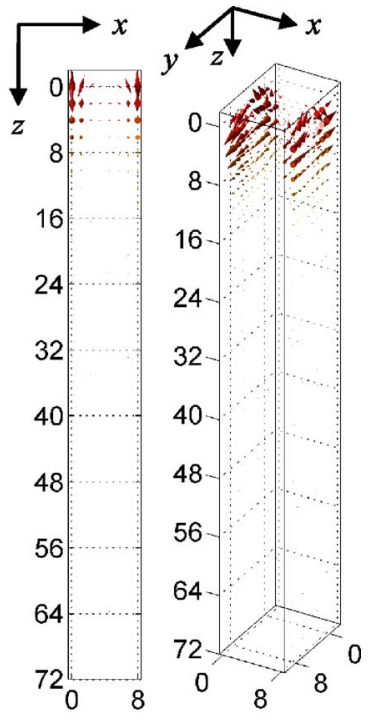

(b)

\section{unit: mm}

FIG. 3. (Color online) The 3D displacement fields of eigenmodes. (a) $\mathbf{k}=(\pi / a, 0), f=77 \mathrm{kHz}$ (point $\mathrm{A}$ of Fig. 2); (b) $\mathbf{k}$ $=(\pi / a, 0), f=199 \mathrm{kHz}$ (point B of Fig. 2).

symbol of BAW modes, such as the first and second bands of in-plane and antiplane transverse waves in the $\Gamma X$ direction. In the higher frequency range, there are numerous peaks, but most peak values are very small.

To investigate the modes of acoustic wave detected in the 3D phononic crystal unit cell, the displacement distributions of the eigenmodes are calculated. The calculating setup is the same as Fig. 1(a) but the initial condition is replaced by a wave source. The source generates a wave package of chosen frequency that is the same as the eigenfrequency of the corresponding wave vector. Because both the wave vector and frequency are assigned, the specific eigenmode is excited and the displacement field is recorded to help identify the properties of modes. The displacement distributions of the eigenmodes are plotted in a 3D vector plot. In Fig. 3 and the following $3 \mathrm{D}$ vector figures, the direction of cones is used to indicate the direction of displacement vector and the size reflect the magnitude of vectors. The color of cones changes with the $z$-axis coordinate of particles to help easily distinguish the location. Besides, the unit cell has grid lines in the location of multiples of the lattice constant.

First, two eigenmodes that differ from those of BAW are calculated. The eigenmodes of wave vector $\mathbf{k}=(\pi / a, 0)$ with frequency $f=77 \mathrm{kHz}$ (point $\mathrm{A}$ in Fig. 2) and $f=199 \mathrm{kHz}$ (point B in Fig. 2) are shown in Figs. 3(a) and 3(b). These modes of wave vector $\mathbf{k}=(\pi / a, 0)$ propagate along the $x$ direction in Fig. 3. The wavelength of the first band with $|\mathbf{k}|$ $=\pi / a$ is $2 a$ and thus only a half of the periodic displacement field is shown is one unit cell. The wave in Fig. 3(a) has a main polarization in the sagittal plane, the $x$ - $z$ plane, and the wave in Fig. 3(b) has a main polarization in the $x-y$ plane. The result shows confined displacement fields; the amplitude decays rapidly as the depth increases. Thus, they present a typical property of surface acoustic wave. An interesting 
phenomenon is that the band which the mode in Fig. 3(a) belongs to has a higher velocity than the transverse wave of BAW modes. That means the surface wave is a leakage type, and usually named pseudo surface acoustic wave (PSAW).

In addition, the peaks overlapped on the BAW modes are also analyzed. For example, the eigenmode of wave vector $\mathbf{k}=(\pi / a, 0)$ with frequency $f=43 \mathrm{kHz}$ is calculated and the distribution of displacement field shows a $y$-direction polarization and the amplitude does not decay rapidly within nine lattice constants. This reflects the property of transverse waves, the same with the mode of in-plane transverse bulk waves. Analysis of another mode of wave vector $\mathbf{k}$ $=(\pi / 2 a, 0)$ with frequency $f=84 \mathrm{kHz}$ also shows a rotation field that remains within the depth of the unit cell. Thus, the peaks overlapped on the modes of BAW present the property of BAW.

The analysis shows that the 2D steel/epoxy square phononic crystal with a free surface allows PSAW instead of SAW. The dispersion curves show an important fact that there exists a complete band gap of both BAW and PSAW (SAW) in this phononic crystal. The complete band gaps obtained by both results of 2D and 3D unit cell locate from $90-143 \mathrm{kHz}$ and $154-199 \mathrm{kHz}$. This is the basis on which to design a phononic crystal waveguide of surface waves.

In brief, some properties of using FDTD method in calculating eigenmodes can be drawn. In the lower frequency range of the first band, the acoustic wave has a long wavelength. Thus some PSAW (or SAW) do not develop well because of the finite depth of the unit cell and the absorbing boundary condition. Also, in the case of energy leakage surface waves, the characteristic displacement decays rapidly, and thus sometimes the sensor on the free surface may be insensitive and miss the leakage modes. Second, in the dispersion curves, some eigenmodes calculated from the FDTD program of 3D condition repeat the result from the 2D BAW conditions. Finally, the natural property of FDTD limits the accuracy of result in high-frequency range. Even with imperfections, the FDTD method is a powerful tool to calculate and analyze the eigenmodes, and the accuracy can be raised by increasing the sampling grid size with the improvement of computers.

\section{SURFACE ACOUSTIC WAVES INSIDE A PHONONIC CRYSTAL WAVEGUIDE}

A phononic crystal waveguide is designed by arranging adjacent point defects in a phononic crystal structure. These defects form a continuous region that is free of inclusions and in which acoustic waves are expected to propagate. Currently, most studies focus on BAW in phononic crystal waveguides $^{9-13}$ and the analysis of SAW in waveguides is just started. ${ }^{14}$ In this section, the FDTD method is used to calculate surface acoustic wave propagation inside a phononic waveguide and, further, the bending waveguides are analyzed.

A phononic crystal waveguide of surface acoustic waves is formed based on the complete band gap of surface waves. The acoustic waves in the waveguide cannot penetrate the periodic structure and thus other eigenmodes (the guided

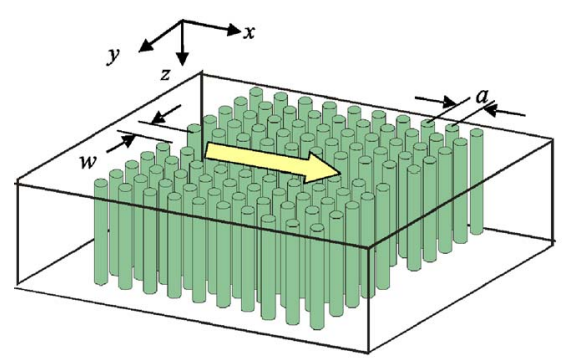

(a)

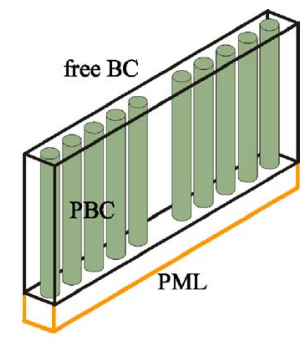

(b)
FIG. 4. (Color online) (a) The structure of a phononic crystal waveguide; (b) the supercell used to analyze the phononic crystal waveguide.

modes) result from the existence of defects that appeared at the range of complete band gaps. In the steel/epoxy phononic crystal presented in Sec. III, the complete band gaps are 90143 and $154-199 \mathrm{kHz}$.

To understand the property of SAW inside phononic crystal waveguides, the supercell technique is used to analyze the dispersion of a waveguide. We consider a waveguide with a $10 \mathrm{~mm}$ width $w$ (the distance between two neighboring cylinders on both sides) as shown in Fig. 4. A 3D supercell consisting of $11 \times 1 \times 9$ unit cells is defined, and then the waveguide is constructed of ten periodic arrayed cylinders and a dislocation in the center with the PBC surrounding it. Similarly, an initial disturbance is defined inside the waveguide, and thus acoustic waves are generated and the displacement field is recorded. Then the eigenmodes are selected from the local maximum peaks of spectra as the procedure of analyzing a unit cell. By setting corresponding boundaries, both the dispersion calculated by $2 \mathrm{D}$ and $3 \mathrm{D}$ supercells are obtained and plotted in Fig. 5. Figure 5 is focused on the frequency of $70-220 \mathrm{kHz}$ to observe defect modes in the range of complete band gaps. The extended bands outside the complete band gaps are omitted and the region is marked in gray. As shown in the result, other defect modes of acoustic waves appear in the range of complete band gaps. The eigenmodes of BAW are marked as solid and hollow circles and those obtained from the 3D supercell are rhombus symbols.

The defect modes of BAW are similar to the result of a previous study, ${ }^{10}$ and there are different defect modes resulting from the free surface and nonreflection condition in the dispersion diagram. In the 3D supercell, sensors on the free surface of phononic crystal waveguide receive the signal of defect modes of both BAW and SAW. The signal of BAW modes has a small peak value and make the spectrum show a complex pattern. Thus, in the 3D waveguide calculation, the BAW modes are removed and only the SAW mode is shown in Fig. 5. Inside the first complete band gap of $90-143 \mathrm{kHz}$, a band extends from wave vector $\mathbf{k}=(\pi / a, 0)$ with frequency $f=87.5 \mathrm{kHz}$ to $\mathbf{k}=(0.05 \pi / a, 0)$ with $f=141 \mathrm{kHz}$, and another band appears from $\mathbf{k}=(0.75 \pi / a, 0)$ with $f=154 \mathrm{kHz}$ to $\mathbf{k}=(0.9 \pi / a, 0)$ with $f=206 \mathrm{kHz}$ in the second complete band gap of $154-199 \mathrm{kHz}$.

The displacement fields of the two defect modes are plotted in 3D vector diagrams as those in Fig. 3. The eigenmodes 


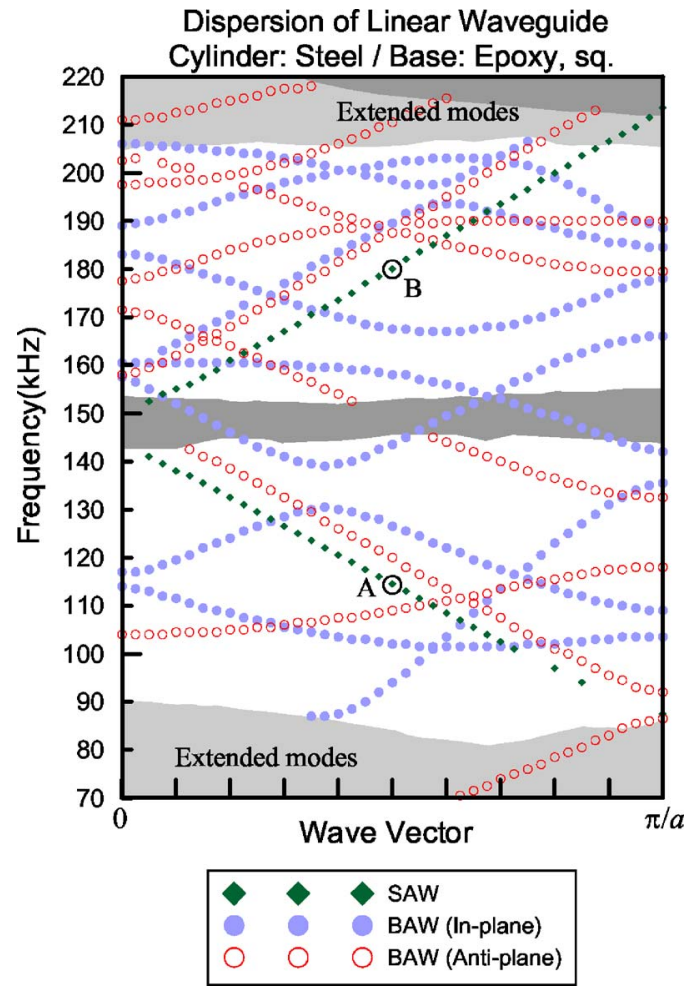

FIG. 5. (Color online) Dispersion diagram of BAW and SAW eigenmodes in the steel/epoxy phononic crystal waveguide with a free surface.

of $\mathbf{k}=(0.5 \pi / a, 0)$ with $f=114.5 \mathrm{kHz}$ (point A in Fig. 5) and $\mathbf{k}=(0.5 \pi / a, 0)$ with $f=180 \mathrm{kHz}$ (point B in Fig. 5) are calculated and shown in Figs. 6(a) and 6(b), respectively. Since the dispersion diagram is presented in a reduced zone scheme, the actual wave vectors of these two modes in folded bands are $\mathbf{k}=(1.5 \pi / a, 0)$ and $\mathbf{k}=(2.5 \pi / a, 0)$ with wavelengths of $1.33 a$ and $0.8 a$, and the consistent result is obtained from the displacement fields of $x-z$ plane. The guided waves propagate along the $x$ direction, and the displacement fields have a confined distribution within the region of waveguides. The maximum amplitude occurs on the free surface and the amplitude decays rapidly in a deeper location. Thus these two bands have a typical property of surface acoustic waves.

A further discussion of the defect modes in phononic crystal waveguide is presented as follows. In the calculation of eigenmode displacement fields, a Gaussian weighted wave package with specified frequency $f$ is set as the source and input finite energy into the supercell structure to excite the selected eigenmode. Then, the sensor on the free surface detects a monotonous displacement variation and the vertical component $U z$ recorded after the wave package is excited. The result shows that the eigenmode has decaying amplitude. This means an energy leakage, partial acoustic waves propagate downward into the half space, and thus the defect modes in this phononic crystal waveguide are actual pseudo surface acoustic waves. Finally, converting the period of signal into propagation distance, the attenuation coefficient is obtained as $-0.049 \mathrm{~dB} / \mathrm{a}$ and $-0.061 \mathrm{~dB} / \mathrm{a}$ for these eigenmodes respectively.
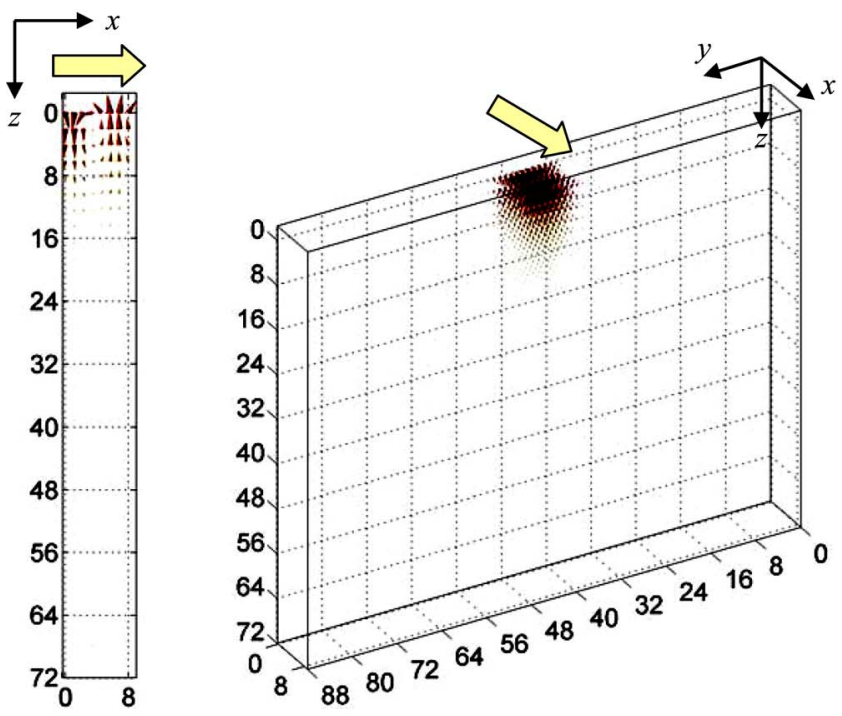

(a)

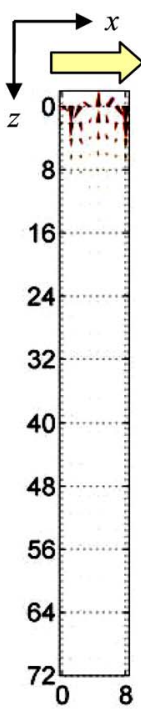

unit: $\mathrm{mm}$

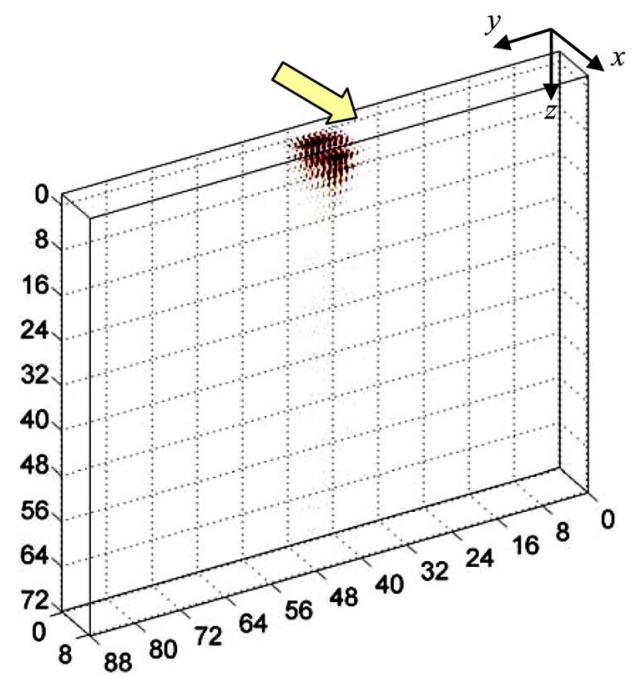

(b)
FIG. 6. (Color online) The 3D displacement fields of defect modes inside the phononic crystal waveguide. (a) $\mathbf{k}=(0.5 \pi / a, 0)$, $f=114.5 \mathrm{kHz}$ (point A of Fig. 5); (b) $\mathbf{k}=(0.5 \pi / a, 0), f=180 \mathrm{kHz}$ (point B of Fig. 5).

To demonstrate the actual wave propagation inside the phononic crystal waveguide, the linear waveguide considered shown in Fig. 7. As a 3D structure, Fig. 7 shows a top view of the free surface and the actual structure extends toward vertically to the surface. The periodic circles present the boundaries of steel cylinders and the epoxy matrix. The waveguide has a width of $10 \mathrm{~mm}$ by removing one row of adjoining cylinder inclusions along the $\Gamma X$-direction, and the length of waveguide is $25 a$. An acoustic wave is generated in the inlet of waveguide with five line sources to simulate an interdigital transducer (IDT). The PSAW defect mode of wavelength $\lambda=1.33 a$ with frequency $f=114.5 \mathrm{kHz}$ is generated and the wave propagation pattern is demonstrated. The displacement field of the $z$-direction component $U z$ is pre- 


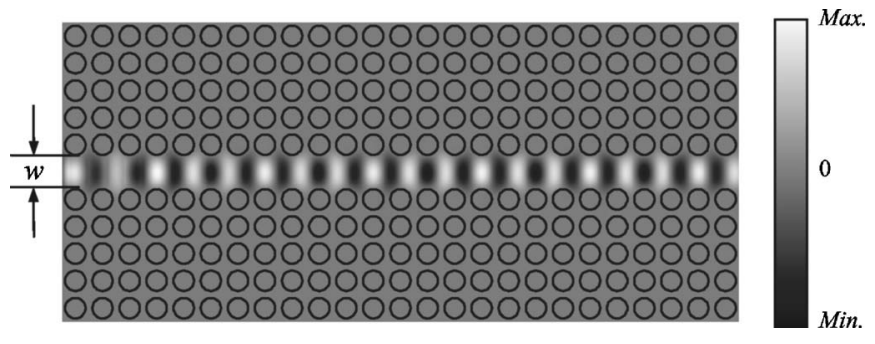

FIG. 7. The $z$-direction displacement field of the $114.5 \mathrm{kHz}$ PSAW inside a linear phononic crystal waveguide with a width of $10 \mathrm{~mm}$.

sented in grayscale to show the surface wave propagation. The black is the minimum (negative) value and the white is the maximum (positive) value. The scale is fixed in this paper for the convenience to compare the amplitude in different cases. The result shows that the PSAW is confined well within the waveguide and only slight energy leaks into the periodic phononic crystal area. In addition, although the PSAW has energy leakage, the PSAW propagates along the waveguide and carries most energy.

In acoustic waveguides, a bend section is an elementary structure to change the wave propagation direction. Here the phononic crystal waveguide with a sharp bend is also calculated to estimate the transmission efficiency. The first case is a simple junction of two $\Gamma X$-direction waveguides. The structure is a $17 a \times 17 a$ area and two $11 a$ waveguides are connected as shown in Fig. 8. The same PSAW of $114.5 \mathrm{kHz}$ defect mode is launched into the waveguide from the inlet in the lower-left corner and the $U z$ displacement field on the surface is shown in the same grayscale. The displacement pattern shows that PASW is still confined within the bending waveguide structure, but most energy of the incident wave is reflected at the corner of the bend. In the case of a linear straight waveguide in Fig. 7, the $114.5 \mathrm{kHz}$ PSAW in the outlet has an amplitude of $2.9 \times 10^{-14} \mathrm{~m}$ as a reference value that results from a monotonous body force of $1 \mathrm{nt} / \mathrm{kg}$ peak value as a wave source. With the identical wave source, the maximum amplitude on the surface of the vertical waveguide branch is about $1.7 \times 10^{-14} \mathrm{~m}(59 \%$ of the reference amplitude) in the bending waveguide. Another phenomenon is that BAW modes are also excited as the wave turns, and thus

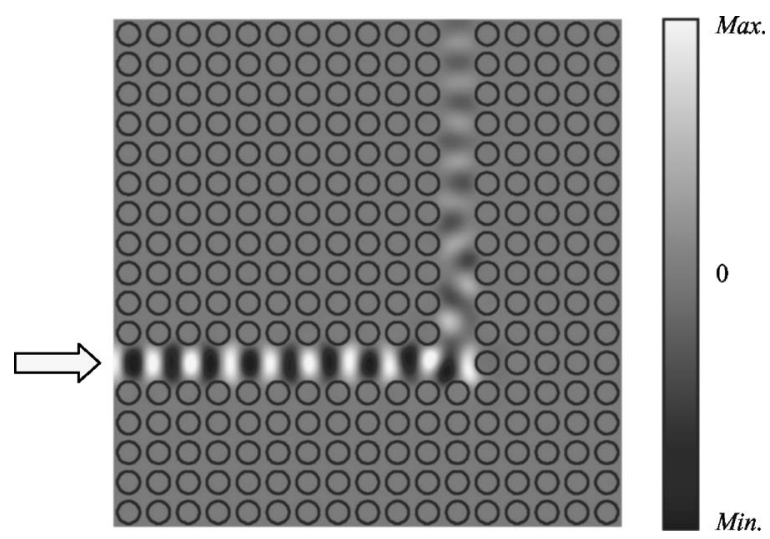

FIG. 8. The $z$-direction displacement field of the $114.5 \mathrm{kHz}$ PSAW inside a sharp bending waveguide.

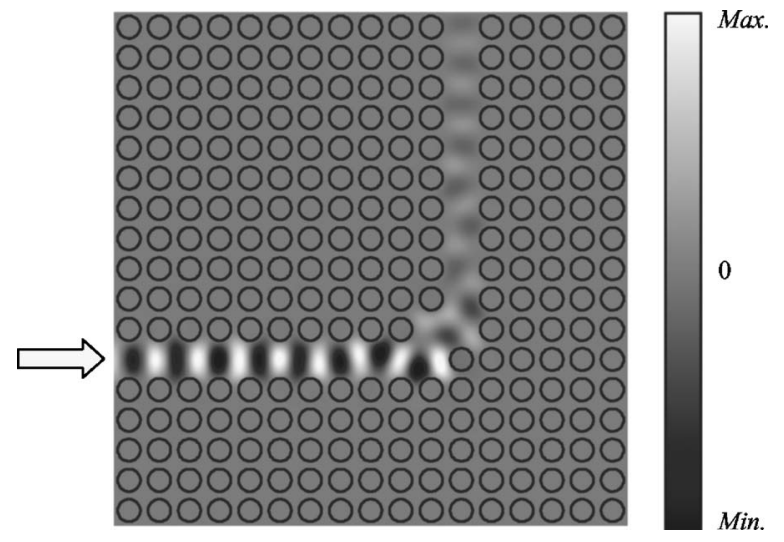

FIG. 9. The $z$-direction displacement field of the $114.5 \mathrm{kHz}$ PSAW inside a three-segment bending waveguide.

considerable energy is taken away and the amplitude in the vertical branch decays rapidly. Thus, a simple joint bend is not an efficient design.

Another bend structure is shown in Fig. 9. It contains two linear waveguides in the $\Gamma X$-direction and one in the $\Gamma M$-direction; thus, the wave turns $45^{\circ}$ twice to the left direction. The $U z$ displacement pattern of $114.5 \mathrm{kHz}$ PSAW defect mode also shows a strong reflection of the incident wave. The amplitude of the turned wave is about 1.5 $\times 10^{-14} \mathrm{~m}(52 \%$ of the reference amplitude $)$ in the $\Gamma M$-direction segment and $0.75 \times 10^{-14} \mathrm{~m}(26 \%)$ in the vertical $\Gamma X$-direction segment. Finally, the mode of the turned wave is rebuilt to a symmetric PSAW mode. In these cases, the wavelengths of PSAW are almost the same with a lattice constant, and thus there is a strong scattering phenomenon when acoustic waves encounter inclusions at their propagating direction. In addition, the antisymmetry conjunction section in Fig. 9 also enhances the scattering and the mode conversion. Therefore, energy disperses while the excited BAW propagates downward, and thus the surface amplitude decays when the PSAW encounters the boundary of the bending waveguides.

To raise the transmission of the bending phononic crystal waveguide, an improved bend structure is introduced. In the study of photonic crystals, the scatters are used to enhance transmission through waveguide bends in two-dimensional photonic crystal slabs. ${ }^{21,22}$ The idea is adopted in this paper to raise the transmission of PSAW in phononic crystal waveguides. The design is based on the prototype shown in Fig. 9 and the cylinders of smaller radius $1.3 \mathrm{~mm}$ are inserted into the $\Gamma M$-direction segment. With the inserted scatters, the conjunction section has a better symmetric geometry property. This also changes the dispersion curve, compared to the result shown in the photonic crystal cases, and results in eigenmodes that propagate in the conjunction section with less loss. In Fig. 10, the distance $d$ between the center of scatters is $16.97 \mathrm{~mm}(3 \sqrt{2} a / 2)$. Then the $U z$ displacement pattern of PSAW of $114.5 \mathrm{kHz}$ is calculated and shown. Obviously, the PSAW passing the corner remains the same eigenmode and therefore the amplitude is raised to 2.1 $\times 10^{-14} \mathrm{~m}$ (72\% of the reference amplitude).

Further calculation shows that the transmission of the guided PSAW is affected by the scatters obviously. If the 


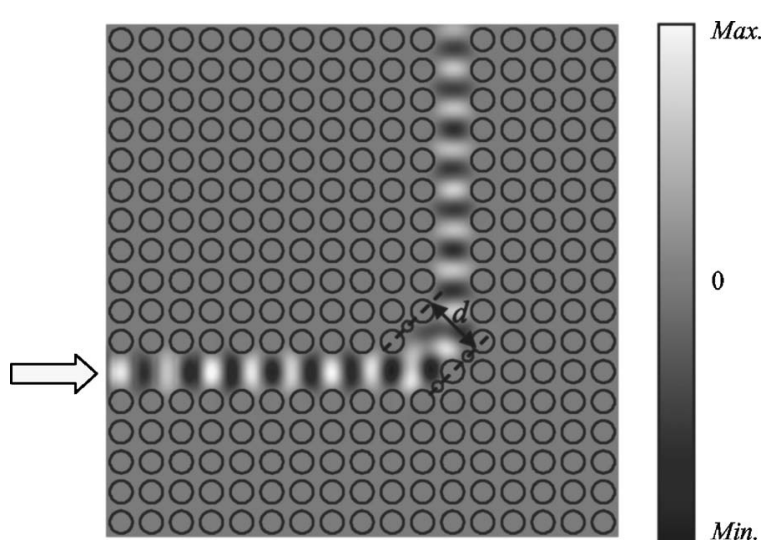

FIG. 10. The $z$-direction displacement pattern of the $114.5 \mathrm{kHz}$ PSAW inside an improved phononic crystal bending waveguide with scatters.

scatters are moved toward the center of the waveguide, the dispersion relation is altered. In the case of the distance $d$ of $11.31 \mathrm{~mm}(\sqrt{2} a)$, the amplitude of turned-PSAW at $114.5 \mathrm{kHz}$ becomes $1.4 \times 10^{-14} \mathrm{~m}(48 \%$ of the reference amplitude). Another important fact is that waves of different frequency in the structure do not have identical performances. The PSAW of $135 \mathrm{kHz}$ in the bend structure of Fig. 10 shifts to BAW mode and the mode decays very rapidly in the turned waveguide, but has a $1.8 \times 10^{-14} \mathrm{~m}(62 \%)$ amplitude ratio in the structure with $d$ of $11.31 \mathrm{~mm}$. Therefore, the transmission efficiency is affected by the scatters and also a function of frequency. Indeed, setting scatters properly in the phononic crystal waveguides can raise the transmission and change the propagation direction in a small region.

\section{CONCLUSION}

In this paper, we implement a FDTD method to analyze propagation of surface acoustic wave inside phononic crystal waveguides. Combing the free surface, PBC, and PML conditions, the eigenmodes of the $2 \mathrm{D}$ phononic crystals with a free surface is calculated in detail. The steel/epoxy phononic crystal shows a complete band gap for both bulk and surface acoustic waves. Further, phononic crystal waveguides are designed based on the complete band gap phenomenon. We found that the defect mode inside the phononic crystal waveguide has an energy leakage because the 3D structure allows the wave propagating downward to the half space. The eigenmodes of the $2 \mathrm{D}$ phononic crystal waveguide with a free surface is thus identified as PSAW. Although the PSAW has a slight energy leakage inside the 2D waveguide, it is possible to have a waveguide with a sharp bend. Adding scatters inside the phononic crystal waveguides can raise the transmission and the design depends on the frequency of PSAW.

\section{ACKNOWLEDGMENT}

The authors gratefully acknowledge the financial support of this research by the National Science Council, Taiwan (Contract No. NSC 94-2212-E-002-040).
*Corresponding author. Email address: wutt@ndt.iam.ntu.edu.tw

${ }^{1}$ M. S. Kushwaha, P. Halevi, L. Dobrzynski, and B. DjafariRouhani, Phys. Rev. Lett. 71, 2022 (1993).

${ }^{2}$ M. S. Kushwaha, P. Halevi, G. Martinez, L. Dobrzynski, and B. Djafari-Rouhani, Phys. Rev. B 49, 2313 (1994).

${ }^{3}$ M. S. Kushwaha and P. Halevi, Appl. Phys. Lett. 64, 1085 (1994).

${ }^{4}$ Y. Tanaka and S. I. Tamura, Phys. Rev. B 58, 7958 (1998).

${ }^{5}$ Y. Tanaka and S. I. Tamura, Phys. Rev. B 60, 13294 (1999).

${ }^{6}$ T.-T. Wu, Z.-G. Huang, and S. Lin, Phys. Rev. B 69, 094301 (2004)

${ }^{7}$ T.-T Wu, Z.-C. Hsu, and Z.-G. Huang, Phys. Rev. B 71, 064303 (2005).

${ }^{8}$ V. Laude, M. Wilm, S. Benchabane, and A. Khelif, Phys. Rev. E 71, 036607 (2005).

${ }^{9}$ M. Kafesaki, M. M. Sigalas, and N. García, Phys. Rev. Lett. 85, 4044 (2000).

${ }^{10}$ A. Khelif, B. Djafari-Rouhani, J. O. Vasseur, and P. A. Deymier, Phys. Rev. B 68, 024302 (2003).

${ }^{11}$ J.-H. Sun and T.-T. Wu, Phys. Rev. B 71, 174303 (2005).
${ }^{12}$ A. Khelif, B. Djafari-Rouhani, J. O. Vasseur, P. A. Deymier, Ph. Lambin, and L. Dobrzynski, Phys. Rev. B 65, 174308 (2002).

${ }^{13}$ Y. Pennec, B. Djafari-Rouhani, J. O. Vasseur, A. Khelif, and P. A. Deymier, Phys. Rev. E 69, 046608 (2004).

${ }^{14}$ Y. Tanaka and S. I. Tamura, International Symposium on Mechanical Wave in Solids, No. 52, 2006 (unpublished).

${ }^{15}$ J.-H. Sun and T.-T. Wu, Proc.-IEEE Ultrason. Symp. 2005, 73 (2005).

${ }^{16}$ Y. Tanaka, Y. Tomoyasu, and S. I. Tamura, Phys. Rev. B 62, 7387 (2000).

${ }^{17}$ P.-F. Hsieh, T.-T. Wu and J.-H. Sun, IEEE Trans. Ultrason. Ferroelectr. Freq. Control 53, 148 (2006).

${ }^{18}$ J. Berenger, J. Comput. Phys. 144, 185 (1994).

${ }^{19}$ W. C. Chew and Q. H. Liu, J. Comput. Acoust. 4, 341 (1996).

${ }^{20}$ F. Chagla, C. Cabani, and P. M. Smith, Proc.-IEEE Ultrason. Symp. 2004, 517 (2004).

${ }^{21}$ A. Talneau, L. Le Gouezigou, N. Bouadma, M. Kafesaki, C. M. Soukoulis and M. Agio, Appl. Phys. Lett. 80, 547 (2002).

${ }^{22}$ A. Chutinan, M. Okano, and S. Noda, Appl. Phys. Lett. 80, 1698 (2002). 